\title{
Common mode noise propagation and effects in a 4-wheel drive electric vehicle
}

\author{
I.Echeverria, F.Arteche, Member, IEEE, M.Iglesias, A.Pradas, J.Piedrafita and F.J.Arcega, Senior \\ Member, IEEE
}

\begin{abstract}
New topologies of electric car use high power switching devices which must be integrated with a growing range of electronic systems in small places. A good performance of the car relies on the electromagnetic compatibility among these systems, which requires the characterization of the noise and interferences present in the system. To characterize the noise, both emission and susceptibility issues as well as coupling mechanisms must be studied. This paper presents the work performed for the electromagnetic mapping of a 4-wheel fully electric car, which involves both simulation and experimental measurements. It has been carried out within the E-VECTOORC project (FP7-INFSO-284708), in collaboration with car manufacturers such as Jaguar Land Rover and Škoda.
\end{abstract}

Index Terms-electric vehicle, electromagnetic compatibility, noise measurement, electromagnetic fields

\section{INTRODUCTION}

$\mathrm{T}$ HE progressive electrification of the car has brought about a growing use of electronics on board the vehicles to replace former mechanical devices as well as enhance the safety and driving conditions. But this intense presence of electrical components is forcing designers to integrate sensing and monitoring elements with high power distribution systems and switching devices such as power converters, which are an essential part of the incoming topologies of electric cars.

This however raises new problems since power converters are a critical source of electromagnetic noise [1][2]. The switching mode of these components may increase the current harmonic content in frequencies over the $\mathrm{kHz}$ [3], which may in turn reach sensitive elements such as control boards of sensing equipment installed on the car throughout the electric layout. Furthermore, the car circuit can form loops in which the conducted noise may cause radiated noise and affect external systems in the infrastructure such as communications and signaling devices [4][5][6]. But the on-board systems can also be affected by external noise coming from neighboring devices as radiated fields. The presence of coupling phenomena might add noise to the system which could compromise the safety and performance of the vehicle.

Because of these issues, more efforts are devoted to guarantee a safe and optimal performance of the car and, at the same time, the nearby systems are protected. The European EMC Directive (2004/104/EC) [7] provides a framework to regulate emissions of vehicles as well as onboard components.
However, this regulation is limited in the frequency domain to radiated emissions of electric field from $30 \mathrm{MHz}$ to $1 \mathrm{GHz}$. Methods for conducted emissions measurement can be found in CISPR 25 standard [8], but these are restricted to component level. In the case of immunity, a set of methods are provided by the ISO series (ISO 11451, ISO 11452). Though these methods can be used as a benchmark for car electromagnetic robustness, they may not provide enough information for the noise characterization since coupling mechanisms and noise propagation issues are not directly assessed. This forces manufacturers to eventually develop their own requirements and testing procedures, which are specific for the electric car scenario and meet their needs for product safety and performance. To do so, the electromagnetic map of the vehicle plays a very important role.

This map is of the utmost importance especially for designers, as it helps to identify the critical areas in terms of emissions, the most susceptible parts of the car and the predominant characteristics of the interferences present in the system. To obtain this map, the emission sources and victim circuits must be identified, as well as the coupling paths between them. For this identification, a component level study is especially helpful before analyzing the complete system, since it provides useful information about the main sources and the characteristics of the noise. This approach has been applied in the mapping of very complex scenarios, such as particle detectors [9][10][11], where the noise emission, immunity and propagation issues are analyzed. This paper presents the work performed in a study devoted to the electromagnetic mapping of a 4-wheel drive fully electric vehicle [12][13].

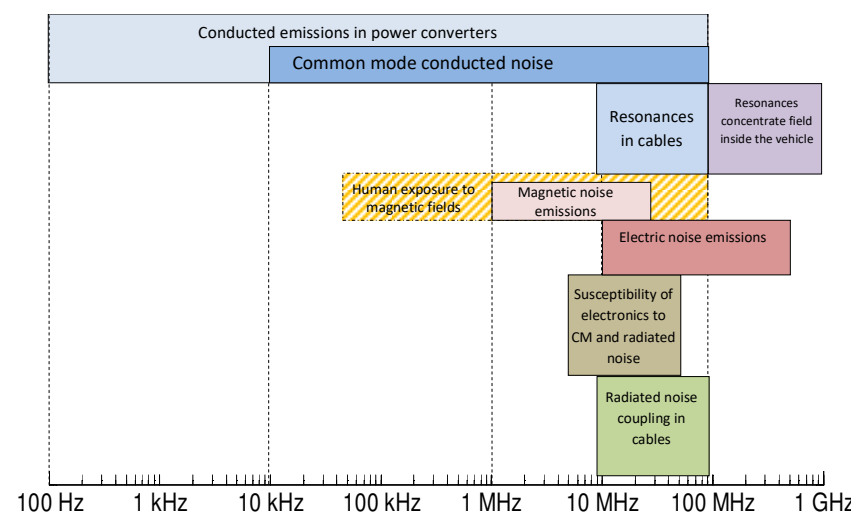

Fig. 1. EMC characterization in electric vehicle 
Fig. 1 shows a compilation of the performed studies in which the electromagnetic interactions identified in the vehicle are represented. The study has been carried out on a vehicle demonstrator of E-VECTOORC, a project in the 7th Framework Programme which has involved car manufacturers such as Jaguar Land Rover and Škoda. The results presented in this paper are focused on the emission characterization, noise propagation in cables and their correlation with the coupling phenomena that takes place in the whole vehicle. After a description of the system under study in section II, the characterization of the conducted noise emissions is presented in section III. Later on, the propagation study on the power cable is exposed in section IV, and the characterization of the radiated interactions is afterwards analyzed. First the radiated emissions are characterized in section $\mathrm{V}$, and then the coupling mechanisms that take place in the car in section VI. Finally, the main conclusions and relevant remarks are presented in section VII.

\section{SYSTEM UNDER STUDY}

The study has been carried out on a 4-wheel drive fully electric vehicle, which has been used as a demonstrator for the E-VECTOORC project. The car and the main information are shown in Fig. 2 and Table I. It consists of a fully electric topology with four switched reluctance (SR) motors each controlled by a power converter. The four converters are fed by a $600 \mathrm{~V}$ link supplied by a battery pack located at the rear of the car. Additionally, a DC/DC power converter is used to adapt the $600 \mathrm{~V}$ battery voltage to the $12 \mathrm{~V}$ line which feeds the on-board electronic equipments. Fig. 3 shows a schematic of the main electric elements that form the car power layout. The control and monitoring of the vehicle is performed through a micro-processor (Autobox) installed at the back. This device is used to set a fixed demanded torque in each motor in a way that a stable speed is achieved during the tests. Measurements required in this study have been taken inside the ITAINNOVA $10 \times 18 \times 7 \mathrm{~m}$ semi-anechoic chamber. Control and monitoring of the car are performed through a USB connection to a laptop located in the control room outside the chamber.

In order to characterize the noise generated by the car, the main emission source must be identified. In this case, the power converters are the critical components since their switching operation contributes to the harmonic content.

\section{CONDUCTED NOISE CHARACTERIZATION}

The switching operation of the power converters used in the car powertrain can turn them into important sources of conducted noise [14][15]. This noise can distinguish two components. One defined as Differential Mode current (DM), which is related to the intrinsic switching conversion process where produced residual high frequency harmonics are not fully filtered by the input/output filters. The other component defined as Common Mode current (CM) finds a coupling path through parasitic elements in nonsymmetrical filters, heat sinks and other devices attached to the power converter case.

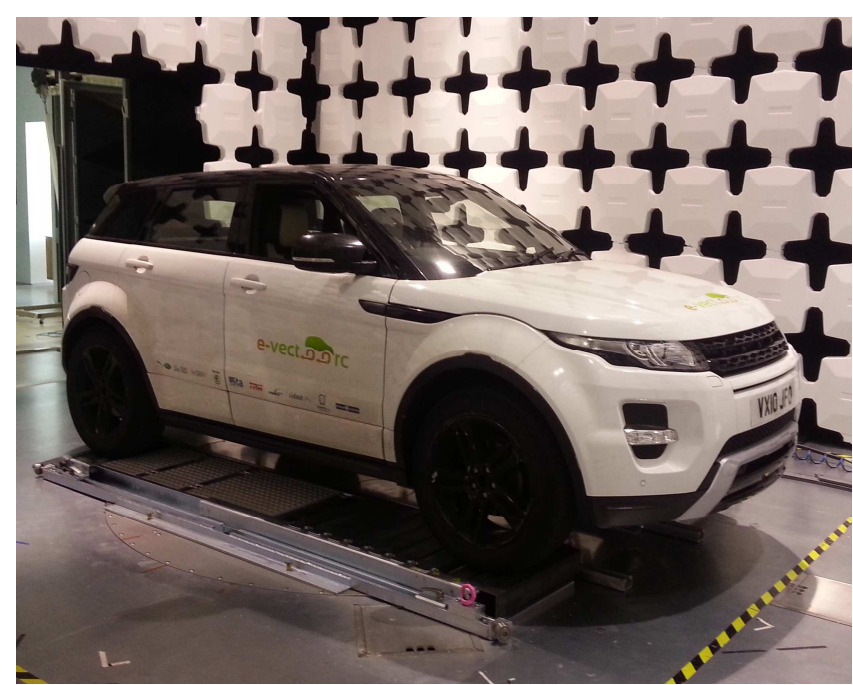

Fig. 2.Vehicle demonstrator

TABLE I

VEHICLE DEMONSTRATOR CHARACTERISTICS

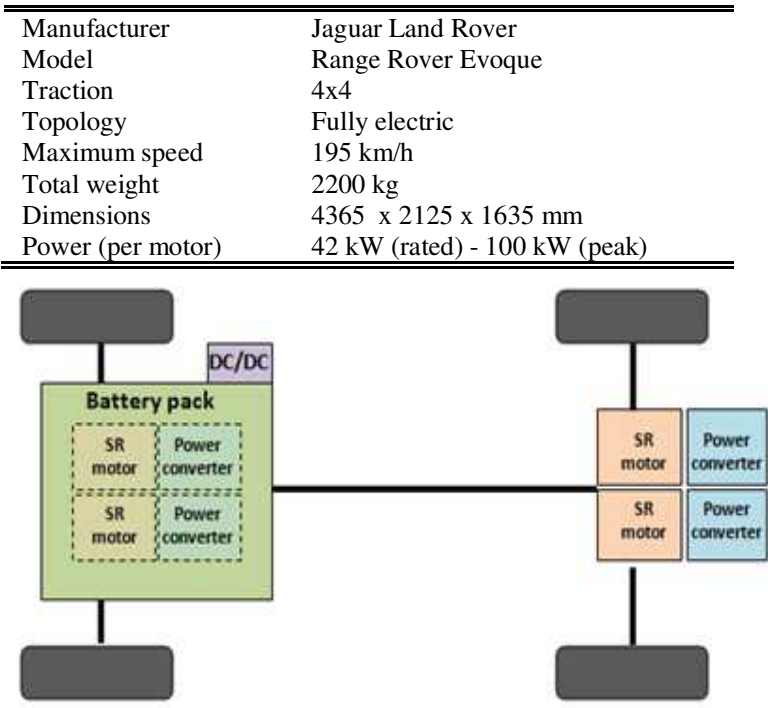

Fig. 3.Vehicle electric layout

In the present section, measurements of both component emissions have been performed on the car while it runs at a fixed speed of $40 \mathrm{~km} / \mathrm{h}$. For these tests, a current clamp has been placed embracing the cables at the DC input in one of the converters (front-right converter is chosen so that the power circuit includes the harnesses that crosses the vehicle). Two measurements have been performed: one with the clamp embracing both terminals, and another with the clamp embracing only one terminal.

The first measurement provides the CM component, while the later provides the sum of both CM and DM components. A direct comparison of both measurements results in the identification of the frequency range in which the $\mathrm{CM}$ component prevails over the DM. This can be seen in Fig. 4, where both $\mathrm{CM}$ and $\mathrm{CM}+\mathrm{DM}$ measurements are displayed. Since both traces are very similar above $10 \mathrm{kHz}$, the graph shows that the conducted noise contains a dominant CM component over this frequency. As a result, the following analysis will be focused on this component. 




Fig. 4.Common mode noise identification

Common mode noise generated by power converters may reach susceptible parts of the system, or produce radiated noise. Both effects can be increased by the cables, which act as propagation paths for the noise in the system. Modeling these elements allows identifying the effect of resonance characteristics and the connections to the system ground on the noise propagation.

\section{NOISE PROPAGATION}

Cables can be modeled using the Multi-Conductor Transmission Lines (MTL) theory [16][17][18]. This approach, in which the cable model is represented in per-unit parameters, assumes transverse electromagnetic (TEM) wave as the propagation mode. The direction of propagation is coincident with the $\mathrm{z}$ axis of the cable rectangular coordinate system [11].

In the MTL theory, a cable with $\mathrm{N}$ inner conductors is represented by the set of $2 \mathrm{~N}$ partial differential equations (1). The complete model of the cable including the shield consists of the inner system representing the central conductors and the inner part of the shield augmented by the coupling effect of voltage and currents flowing through the shield [19]:

$$
\begin{aligned}
& \frac{\partial v(z, t)}{\partial z}=-R i(z, t)-L \frac{\partial i(z, t)}{\partial t}+v_{s h}\left(i_{o}(z, t)\right) \\
& \frac{\partial i(z, t)}{\partial z}=-G v(z, t)-C \frac{\partial v(z, t)}{\partial t}+i_{s h}\left(v_{o}(z, t)\right)
\end{aligned}
$$

where $i(z, t)$ and $v(z, t)$ are $\mathrm{Nx} 1$ vectors representing the current and the voltage with respect to the reference conductor; the parameters L, C, R, G, are the per-unit-length inductance, capacitance, resistance, and conductance $\mathrm{NxN}$ matrices, respectively, containing the cross-sectional dimensions and properties of the line; $\mathrm{z}$ is the position along the transmission line; and denotes the time variable. The generators $v_{s h}\left(i_{o}(z, t) i_{s h}\left(v_{o}(z, t)\right.\right.$ represent the coupling effect in the central conductors of currents flowing through the cable shield and the voltage between the shield and the structure.

In this study, the traction harness that feeds the power converter has been selected, since it runs along the car structure, resulting in long paths which, combined with common mode currents and high power conversion, might present a significant contribution to the radiated noise profile of the vehicle. The harness is composed of two cables, each surrounded by a shield. The geometrical characteristics of the cable are used for the theoretical estimation of the L-C-R parameters [20]. These are shown in eq. (2). For the cable, a $2 \mathrm{~m}$ length is used to represent the case of a front inverter fed by the battery at the back of the vehicle.

$$
\begin{aligned}
& R=\left[\begin{array}{cc}
0.00034 \cdot\left(1+\sqrt{\frac{f}{1000}}\right)+0.00082 \cdot\left(1+\sqrt{\frac{f}{450}}\right) & 0.00082 \cdot\left(1+\sqrt{\frac{f}{450}}\right) \\
0.00082 \cdot\left(1+\sqrt{\frac{f}{450}}\right) & 0.00034 \cdot\left(1+\sqrt{\frac{f}{1000}}\right)+0.00082 \cdot\left(1+\sqrt{\frac{f}{450}}\right)
\end{array}\right] \frac{\Omega}{m} \\
& C=\left[\begin{array}{cc}
1.52 \cdot 10^{-10} & 0 \\
0 & 1.52 \cdot 10^{-10}
\end{array}\right] \frac{F}{m} \quad L=\left[\begin{array}{cc}
3.5 \cdot 10^{-7} & 0 \\
0 & 3.5 \cdot 10^{-7}
\end{array}\right] \frac{H}{m}
\end{aligned}
$$

Common mode noise is directly injected by connecting the two conductors at one end of the cable to a voltage source, in a way that an interfering current is forced to flow through both terminals. At the other end, each terminal is connected to a $\mathrm{ZL}$ impedance, which is introduced in the model as a generalized Thevenin equivalent. Two load conditions are analyzed: first, a balance load in which each terminal is connected to $150 \Omega$ ( $150 \Omega$ is used as the normalized impedance for studies in CM propagation in cables); a second case represents an unbalance load in which one terminal is connected to a $150 \Omega$ load and the other terminal is connected to ground.

Since the R, L, C, G parameters are directly related to the cable geometry, deviations in the conductor position may result in parameter variations which must be also taken into account. This is achieved by applying stochastic methods in the assessment which account for geometry deviations. In this approach, a Monte Carlo method [21] is applied in a way that a batch of 100 samples is simulated.

As the attenuation in the signal along the cable pays an important role, this can be analysed with the transfer function in eq. (3), which relates the common mode currents at the end of the cable $(\mathrm{z}=\mathrm{L})$ with the common mode currents at the beginning $(\mathrm{z}=0)$.

$$
T F_{I c m}=\frac{I_{c m}(L)}{I_{c m}(0)}
$$

Fig. 5 shows the transfer function profile for both load conditions. As it can be seen, the balance case attenuates the currents at high frequencies, while an unbalanced situation would results into an amplification of the common mode resonances over $10 \mathrm{MHz}$. These results show the impact that the impedance at the end of the power cables has on the noise, in a way that low impedances like the presence of capacitances used in filters may cause higher resonances, resulting in noise amplification at certain frequencies. As the common mode is strongly related to the radiating interactions of the car, the characterization of the radiated noise emissions and coupling capacity of the whole system has been analyzed. 


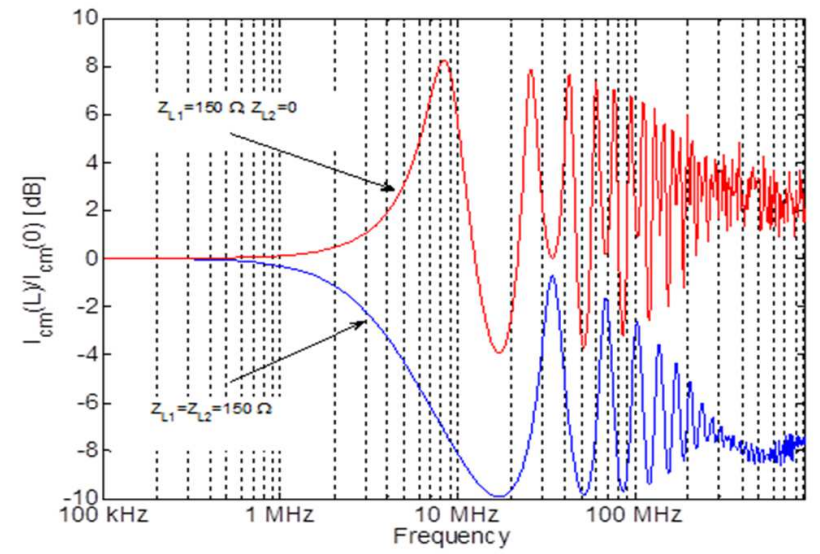

Fig. 5.Common mode current transfer function in traction cables

\section{RADIATED EMISSION CHARACTERIZATION}

The car radiated emissions have been measured with a set of antennas (Fig. 6 and Fig. 7 show some of the antennas used) covering the low and high frequency ranges. The antennas have been placed $3 \mathrm{~m}$ from the vehicle (clause 5.2.3 of CISPR 12 standard), and both lateral and frontal sides of the car have been measured. For the magnetic field testing, a loop antenna $(10 \mathrm{kHz}-30 \mathrm{MHz})$ has been used. Electric field has been measured with a dipole antenna in low frequency range $(30 \mathrm{~Hz}-50 \mathrm{MHz})$ and a log-periodic antenna in high frequency range $(30 \mathrm{MHz}-2 \mathrm{GHz})$ in both vertical and horizontal polarizations. Tests have been performed with the car in standby mode (motors powered but not driving traction), and traction mode (vehicle running on free coupling rolls at steady speed of $40 \mathrm{~km} / \mathrm{h}$, as defined in clause 5.3 of CISPR 12 standard).

Fig. 8 shows the spectrum profile of the magnetic field emissions as measured in both front and sideways positions. The graph also displays the measurement with the vehicle completely switched off for reference. The measured spectrum shows a rather low content of noise, with the main contribution over $10 \mathrm{MHz}$. This involves a correct grounding and shield connection strategies in the power cables which bring a reduction in the radiating effects of the common mode noise component, as it was shown in the emission studies performed at component level in [12].



Fig. 6.Electric field measuring with log-periodic antenna



Fig. 7.Magnetic field measuring with loop antenna

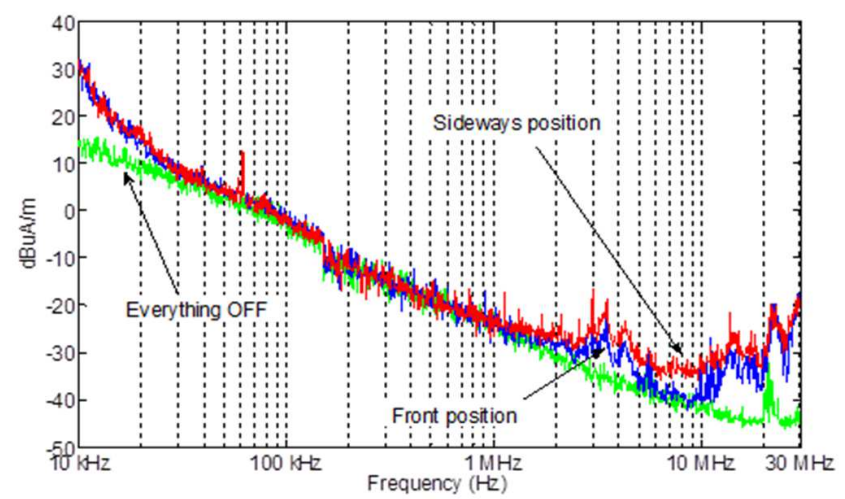

Fig. 8. Magnetic field emissions

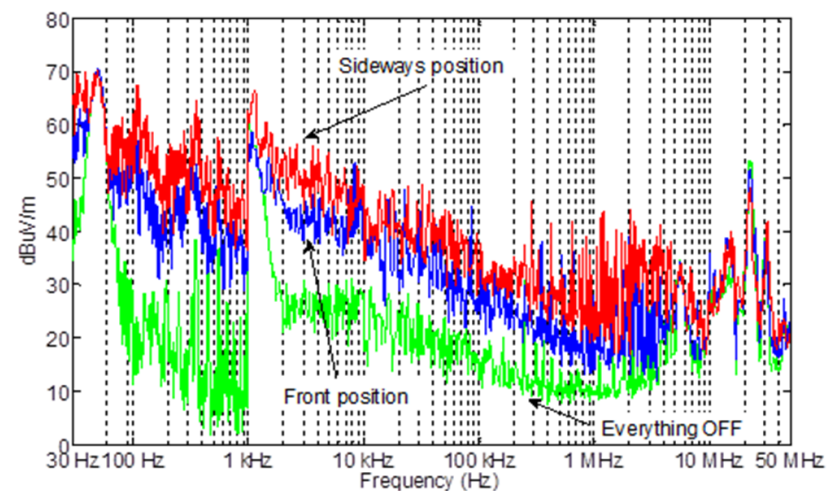

Fig. 9. Electric field emissions (low frequency)

Fig. 9 shows the electric field emission in the low frequency range, and Fig. 10 shows the electric field emission in the high frequency range for horizontal polarization (a similar profile is obtained in vertical polarization). Limits are defined in standards (ECE R10) over $30 \mathrm{MHz}$ for average and quasi-peak detector. Though the measurements have been taken with maximum detector, these limits are included in the plot as a reference. The results show that emissions are slightly lower when the antenna is placed in frontal position below $10 \mathrm{MHz}$, however similar profiles are found in higher frequencies. It can also be observed that the harmonic content in the electric field range is significantly higher over the reference static mode when compared with the magnetic field spectra, which suggests a predominantly electric behaviour of the car. 


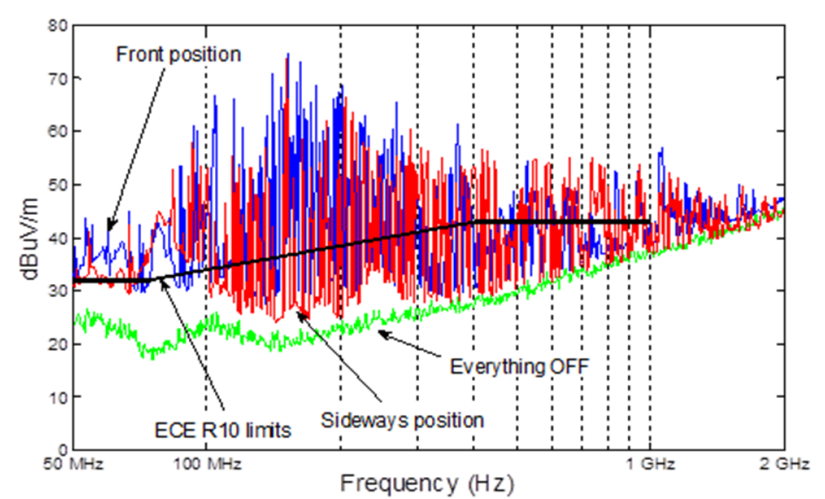

Fig. 10. Electric field emissions (high frequency) - Horizontal polarization

\section{RADIATED NOISE COUPLING}

Incidental radiated noise coming from the environment external to the car involves a source of interferences that might induce disturbances inside the vehicle. These disturbances may come typically as induced currents in the cables due to the coupling mechanisms that take place when the incoming field reaches the frame of the car. The currents induced may flow along the metallic enclosures and produce inner fields that might induce currents as well in the cables. To analyze this phenomenon, a radiated coupling test has been performed at system level on the car.

Coupling effects of external fields are usually assessed with transfer functions [22] that relate the incidental field measured at a fixed point with the induced current in a cable, as it is shown by eq. (4).

$$
T F=\frac{\text { current in cable }(m A)}{\text { radiated field level }(V / m)}
$$

To perform the tests, a radiated electric field has been injected at different frequencies selected along the range under study (from $10 \mathrm{kHz}$ up to $400 \mathrm{MHz}$ ). The radiated field has been produced with an antenna connected to a signal generator (Fig. 11 shows one of the injecting antennas placed in front of the car). A current clamp is used to measure the induced current (a signal-to-noise ratio -SNR- is extracted to obtain the real contribution of the field).

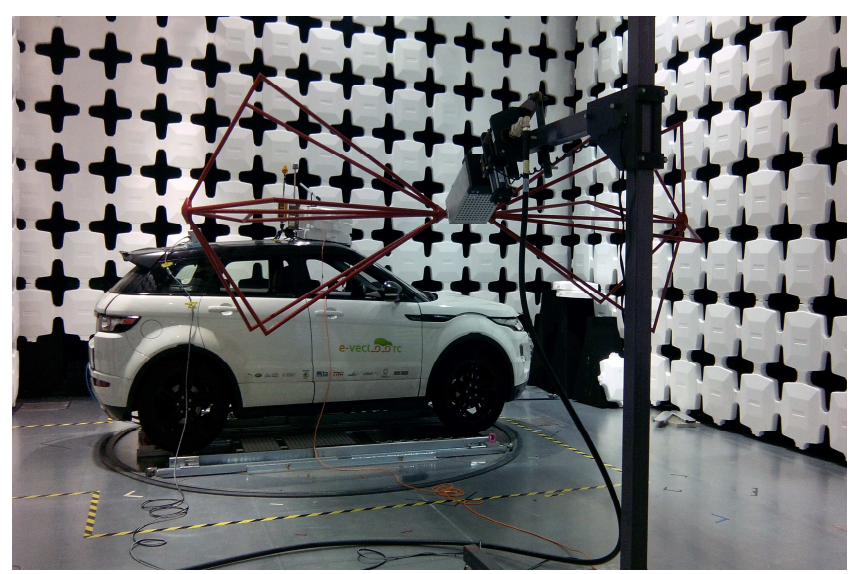

Fig. 11. Radiated noise injection

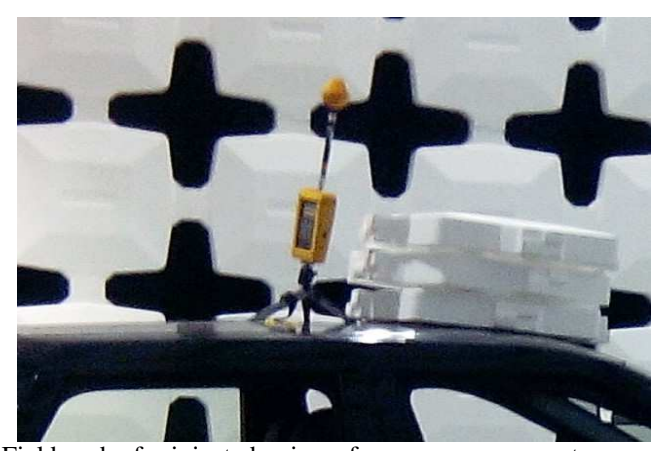

Fig. 12. Field probe for injected noise reference measurement

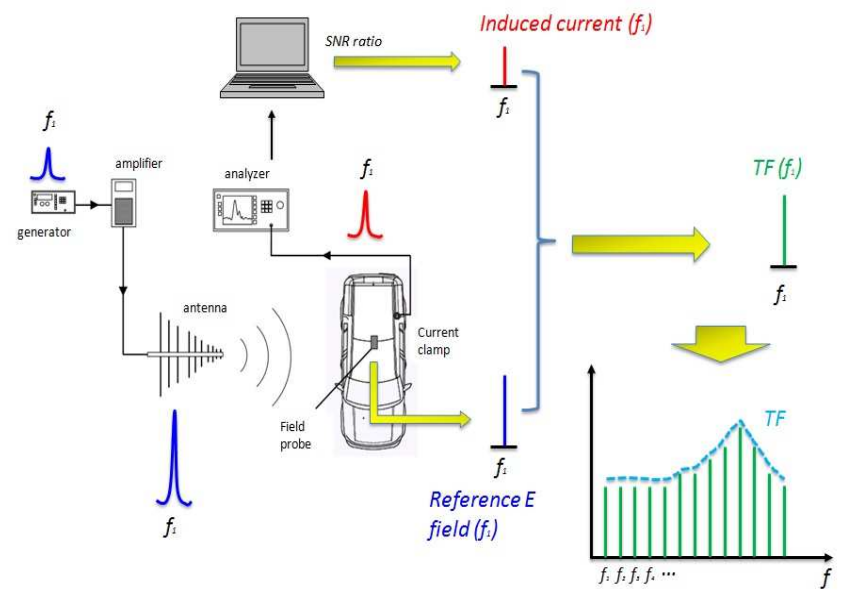

Fig. 13. Noise coupling characterization

At the same time, the radiated field that reaches the car is measured with an E-field probe located on the roof (Fig. 12). The measuring process is represented in Fig. 13. If the transfer function in eq. (4) is computed for each injected frequency, the transfer function frequency profile can be obtained for the whole frequency set in one measuring point of the vehicle. A total of five points have been selected over the car electric layout, in a way that both power and signal cables are assessed. These points are shown in Fig. 14.

In the following, the coupling transfer function at each point has been obtained for both horizontal and vertical injected fields. This provides a set of functions that represent the coupling characteristics of the radiated noise reaching the car. These functions are shown in Fig. 15 and Fig. 16 (horizontal and vertical polarization respectively).

In each point, the common mode noise component has been measured and used in eq. (4), so the coupling functions represent the way radiated noise couples common mode noise in the car. The results show a similar behavior among the tested points, especially at higher frequencies. At low frequencies, point P4 show lower coupling levels in both polarities, which can be explained as it is a signaling cable and, therefore, has high impedance.

This point belongs to a shorter cable in comparison with the rest of the points, and its location lies close to the vehicle ground plane. Besides, this cable runs in the rear next to the battery pack, which together with the boot structure provides higher shielding. All these features contribute to a reduction in the cable coupling capacity, as the plot shows. 




Fig. 14.Points for induced noise measurement

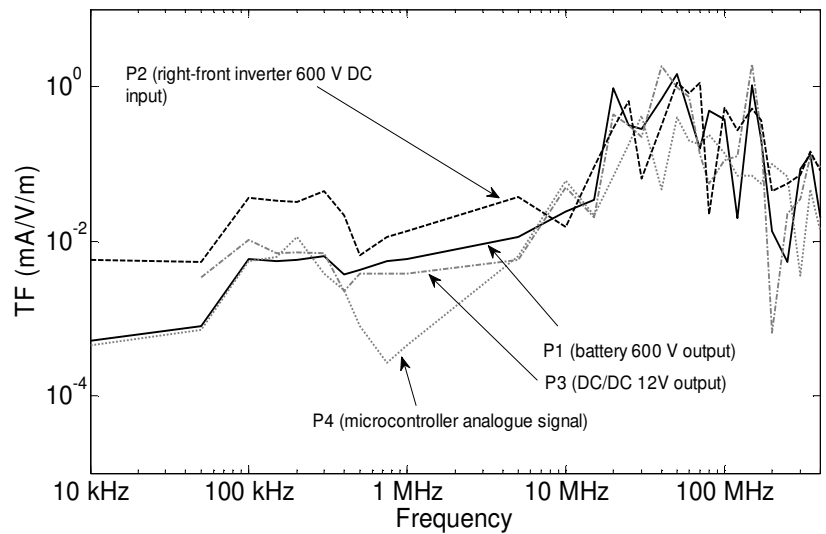

Fig. 15. CM coupling transfer functions (horizontal polarization)

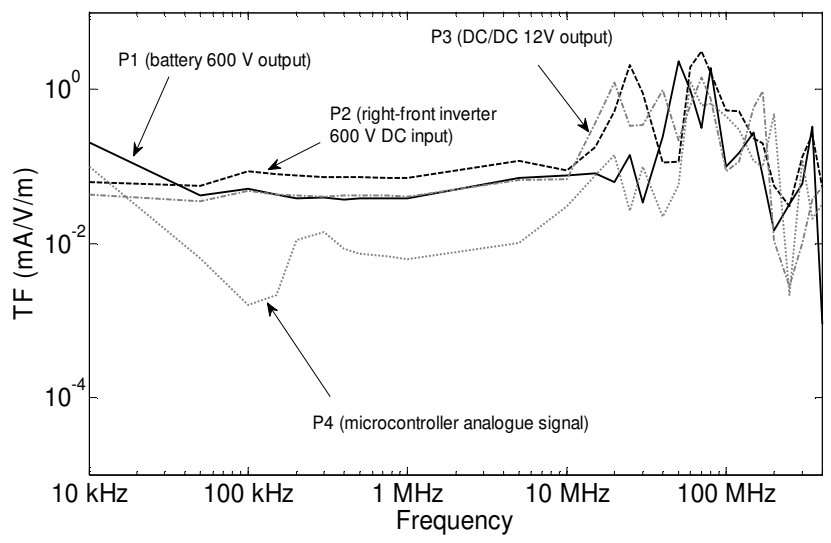

Fig. 16. CM coupling transfer functions (vertical polarization)

In the case of the cable measured at the $12 \mathrm{~V}$ output of the DC/DC (P3), a significant increase in the coupling is observed when switching from horizontal to vertical polarization. This shows the influence of the cable routing, since the cable runs along the vehicle from rear to front connecting the DC/DC converter (located in the back) with the $12 \mathrm{~V}$ car battery (located in the front).

A similar effect can be found in pint $\mathrm{P} 2$, which corresponds to the power cable at the $600 \mathrm{~V}$ input of the right front converter. This cable, as well as in P3, runs along the vehicle length connecting the battery pack in the rear with the converter in the front. In this case the coupling level is higher in comparison with $\mathrm{P} 2$ which belongs to the DC power network that connects the four converters in the vehicle. This increases the circuit area and length, resulting in a higher coupling capacity. In fact, coupling noise can be seen as a current source which depends on the incident field and the circuit geometry. In this case, the overall contribution at the DC input of the inverter is higher, producing higher levels of induced current (as it can be seen in both polarities).

Point P1 corresponds to the $600 \mathrm{~V}$ output of the battery pack. While this belongs to the same electric point than P2 and gets also affected by the polarization of the incident field, the length of the cable is shorter and its location limited to the back part of the vehicle. As a result, the coupled noise in this point is lower in comparison with $\mathrm{P} 2$.

A global analysis of the results reveals a lower level of the coupling transfer function in the points that are located in the rear of the car, which suggests that this part of the vehicle provides a higher shielding in lower frequencies when compared with the front part. If the average value of the transfer function is computed for each polarization over the 6 points under study, the plot in Fig. 17 is obtained. As the plot shows, the coupling mechanism is higher in frequencies above $10 \mathrm{MHz}$, which correlates with the results obtained in Section IV in which resonances of common mode currents were observed above this frequency. A higher capacity of the wire to amplify the common mode currents involves also a higher capacity to receive noise as common mode currents. This leads to the conclusion that the common mode path of the currents in the car layout drives the noise propagation especially over $10 \mathrm{MHz}$.

The measured signal spectrum in emission tests contains the narrow band noise produced by the vehicle, considered as an uncontrolled noise source. On the other hand, radiated field injected into the car may involve a broadband spectrum produced by a controlled generator as well as ambient sources. However, in the case of the coupling tests the field is generated at one specific frequency in a way that a sufficiently high signal-to-noise ratio is obtained in the cable. Therefore the spectrum can be analyzed as a narrow band signal.

Broadband spectra coming from other ambient sources are also avoided since the tests are performed within an isolated semi-anechoic chamber. As a result, the narrowband profile obtained in the coupling tests can be compared with the narrowband profile in the emission spectra in order to compare the phenomena observed in the radiated coupling tests with the emission capacity of the car.

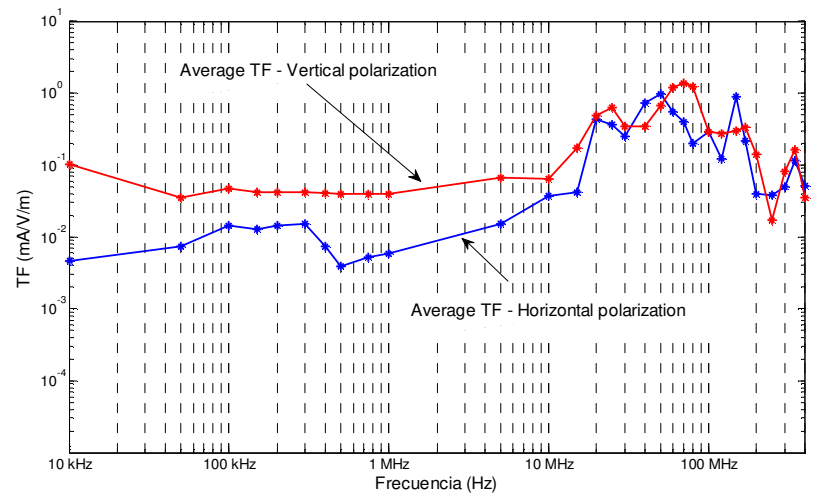

Fig. 17. Average $\mathrm{CM}$ transfer function for horizontal and vertical polarizations 




Fig. 18.Emission-coupling comparison (horizontal polarization)

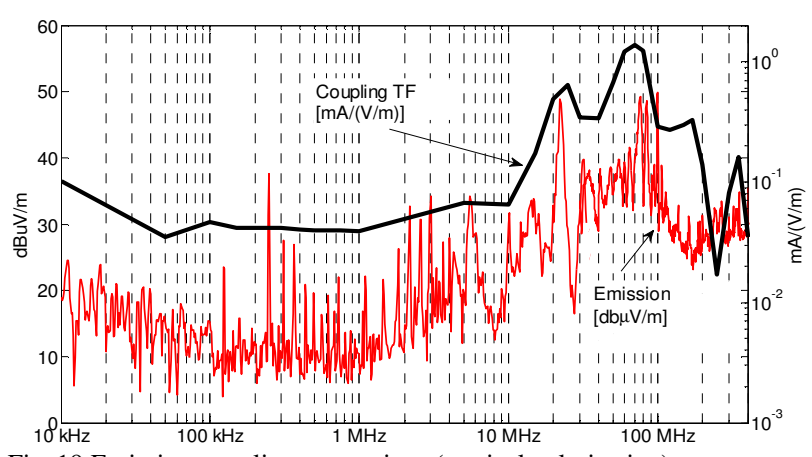

Fig. 19.Emission-coupling comparison (vertical polarization)

Fig. 18 and Fig. 19 show the stand-by radiated emissions in the frequency range tested for coupling with both horizontal and vertical polarizations respectively. On the graphs, the obtained coupling transfer functions are also plotted in order to compare the shape of the functions. As it can be seen, the coupling factor increases along with the emissions, reaching in both cases its maximum between $20 \mathrm{MHz}$ and $100 \mathrm{MHz}$, which illustrates that the emission efficiency of the car matches the coupling profile in the same way an antenna emits with more efficiency in the frequency range where the receiving efficiency is higher.

\section{CONCLUSIONS}

The work presented in this paper has provided a way to characterize the noise present in a high power electric car distribution system. The results obtained throughout the analysis reveal that common mode currents play a very important role in the noise interactions of the car.

First, a conducted noise study has shown the emission profile of the most critical component, that is, the power converter. Here, the switching process contributes with a significant harmonic content in the common mode component of the currents within the power cables. Common mode currents might be an important source of radiated noise when the path they follow includes loops and ground returns. Besides, the impedance of the cables may increase the resonance effects of common mode currents when capacitive elements are used, which involve low impedances at high frequencies, as the MTL models have shown in this study.

Those frequency ranges in which resonances occur may be related to a higher capacity of the cable to couple noise. This has been observed in the results of a radiated coupling test, in which the coupling transfer function of the whole car in different points of the electric layout reveal a higher capacity for external radiation to induce noise in frequencies over 10 $\mathrm{MHz}$. When comparing the coupling results with the emission spectra, similarities have been detected in the $10 \mathrm{kHz}-400 \mathrm{MHz}$ profile, in which the coupling mechanism grows towards the $80 \mathrm{MHz}$ at the same time the electric field emission. This illustrates the geometrical effect of the electrical layout on the electromagnetic interactions of the car, since a dual behaviour emitter-receiver takes place.

The results exposed herein, as part of a detailed electromagnetic mapping of the vehicle, offer information which can be used by designers to increase the robustness of the car, enhance the safety and optimize the performance once the critical parts and relevant noise interactions are identified.

\section{ACKNOWLEDGMENT}

The authors are grateful for the support of other members of the E-VECTOORC project consortium. The research leading to these results has received funding from the European Union's Seventh Framework Programme FP7/2007-2013 under grant agreement $\mathrm{n}^{\circ} 284708$.

\section{REFERENCES}

[1] S. Guttowski, S. Weber, E. Hoene, W. John and H. Reichl, "EMC issues in cars with electric drives," Proc. IEEEInternational Symposium on Electromagnetic Compatibility, pp.777-782 vol.2, Aug. 18-22, 2003.

[2] G.L Skibinski, R.J. Kerkman and D. Schlegel, "EMI emissions of modern PWM AC drives," IEEE in Industry Applications Magazine, vol.5, no.6, pp.47-80, Nov/Dec 1999.

[3] Q. Xiaohui, L. Xiaoquan, Z. Yang, D. Yinghua, J. Ningqiu, R. Rong and Y. Qiutong, "Conductive EMI noise analysis for large power switched reluctance machine," Proc. 2010 IEEE in Electric Power and Energy Conference (EPEC), pp.1-4, Aug. 25-27, 2010.

[4] F. Silva and M. Aragon, "Electromagnetic interferences from electric/hybrid vehicles," Proc. 2011 URSI General Assembly and Scientific Symposium, pp.1-4, Aug. 13-20, 2011.

[5] M. Dong, L. Zhai, R. Gao and X. Zhang, "Research on radiated electromagnetic interference (EMI) from power cables of a threephase inverter for electric vehicles," Proc. 2014 IEEE Conference and Expo in Transportation Electrification Asia-Pacific (ITEC AsiaPacific), pp.1-5, Aug. 31 2014-Sept. 3, 2014.

[6] D. Mansson, R. Thottappillil, M. Backstrom and O. Lunden, "Vulnerability of European Rail Traffic Management System to Radiated Intentional EMI," IEEE Transactions on Electromagnetic Compatibility, vol. 50, no. 1, pp. 101-109, Feb. 2008.

[7] European Commission Directive 2004/104/EC of 14 October, 2004.

[8] EN 55025:2009 (CISPR-25) - Vehicles, boats and internal combustion engines. Radio disturbance characteristics. Limits and methods of measurement for the protection of on-board receivers, April 2009.

[9] F. Arteche, C. Esteban, I. Echeverria, M. Iglesias, C. Rivetta and I. Vila, "Global noise studies for CMS Tracker upgrade," Ref. JINST 5 C12029 - Journal of Instrumentation, vol.5, p. 6, 2010.

[10] F. Arteche, C. Esteban, M. Iglesias, A. Gimeno, F.J. Arcega, M. Johnson and W. Cooper, "Power Network impedance effects on noise emission of DC-DC converters," Ref. JINST 7 C01045 - Journal of Instrumentation, vol.7, p. 8, 2012.

[11] F. Arteche, EMC: a mapping for the CMS experiment, Ph.D. dissertation, Univ. de Oviedo, 2004.

[12] M. Iglesias, I. Echeverria, F. Arteche, J. Piedrafita, A. Pradas, J. De Clercq, "EMC mapping of a power train for fully electric 4-wheel drive vehicle," Proc. 2014 International Symposium on Electromagnetic Compatibility (EMC Europe), pp. 414-419, Sept. 14, 2014.

[13] M. Iglesias, I. Echeverria, F. Arteche, J. Piedrafita, A. Pradas, F.J. Arcega, J. De Smet, "A characterization of EM coupling in a fully electric 4-wheel drive vehicle," Proc. 2015 IEEE International 
Symposium on Electromagnetic Compatibility (EMC), pp.656-661, Aug. 16-22, 2015.

[14] H.Chen, Y.Yan and H.Zhao, "Extraction of Common-Mode Impedance of an Inverter-Fed Induction Motor," IEEE Transactions on Electromagnetic Compatibility, vol. 58, no. 2, pp. 599-606, April 2016.

[15] F. Costa, C. Vollaire and R. Meuret, "Modeling of conducted common mode perturbations in variable-speed drive systems," IEEE Transactions on Electromagnetic Compatibility, vol. 47, no. 4, pp. 10121021, Nov. 2005.

[16] C. R. Paul, Introduction to Electromagnetic Compatibility, Ed. NY: Wiley-Interscience, ISBN-0-471-54927-4, 1992.

[17] C. R. Paul, Analysis of multi-conductor transmission lines, Ed. WileyIEEE Press ISBN-0-471-02080-X, 2007.

[18] F. B. M. Van Horck, A. P. J. Van Deursen and P. C. T. Van der Laan, "Common-mode currents generated by circuits on a PCB-measurements and transmission-line calculations," IEEE Transactions on Electromagnetic Compatibility, vol. 43, no. 4, pp. 608-617, Nov 2001.

[19] L. Jung and J. L. ter Haseborg, "Evaluation of measured complex transfer admittance for the characterization of shield inhomogeneities of Multi-conductor Cables", IEEE Trans. on Electromagnetic Compatibility, vol. 41, Issue 4, pp. 460-468, Nov. 1999.

[20] M. M. Al-Asadi, A. P. Duffy, A. J. Willis, K. Hodge and T. M. Benson, "A simple formula for calculating the frequency-dependent resistance of a round wire," Microw. Opt. Technol. Lett., vol. 19, pp. 84-87, Oct. 1998.

[21] A. Ciccolella and F. G. Canavero, "Stochastic prediction of wire coupling interference," Proc. 1995 IEEE International Symposium on Electromagnetic Compatibility (1995. Symposium Record.), pp.51-56, Aug. 14-18, 1995

[22] M. Zerrer, M. Aidam, W. Koehler and S. Tenbohlen, "The benefit of characteristic transfer functions in automotive EMC," Proc. ISAPE 2008. 8th International Symposium on Antennas, Propagation and EM Theory, pp.933-936, Nov. 2-5, 2008

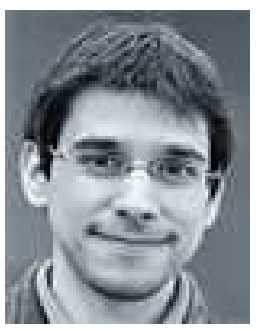

Iván Echeverría received the M.Sc. in Renewable Energy and Energy Efficiency in 2011 and the Ph.D. degree from the University of Zaragoza in 2016. Starting work at ITAINNOVA in 2006, nowadays he belongs to the Power Electric Systems group where he takes part in research projects from different sectors, including automotive and vertical transportation applications. His current research activities are focused on EMC characterization of complex systems and energy efficiency studies.

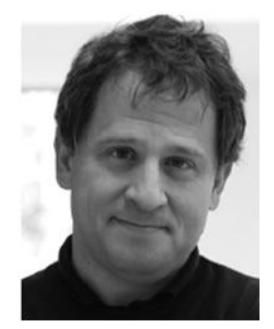

Fernando Arteche received the Laurea and $\mathrm{Ph} . \mathrm{D}$. degrees in electrical engineering from the University of Oviedo, Oviedo, Spain, in 1998 and 2004, respectively. From 1999 to 2005, he worked at the European Organization for Nuclear Research (CERN), Geneva, Switzerland. $\mathrm{He}$ was involved in the design of the superconducting magnets for the new particle accelerator and the power supply system of the CMS experiment. From 2005 to 2007, he worked as a Academic Visitor at Imperial College, London, to design Silicon Strip Tracker detector of CMS experiment. He joined Instituto Tecnológico de Aragón in 2007, working on EMC studies and high-efficiency power systems design for complex installations and transport. His current research interests include EMC in HEP detectors, electric vehicles, and regenerative systems based on supercapacitors and batteries.

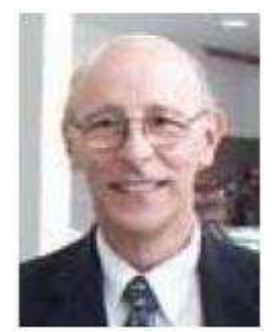

Francisco J. Arcega (M'80-SM'05) received the M.Sc. in Physics in 1976 and $\mathrm{PhD}$ in Physics in 1981 at the University of Zaragoza, Spain. In 1976 he joined the Electronics Department at the University of Zaragoza and since 1982 he is in the Electrical Engineering Department where he is currently Professor (CEU). He is Member of the IEEE since 1980, Senior since 2005 and currently member of the Directive of the Spanish Chapter of the Education Society of the IEEE and Chair of the Chapter (2014-15). His main research interests are in the field of electrical measurements and their applications in the industry field. As well, he is involved with quality in the education and in the laboratory activities.

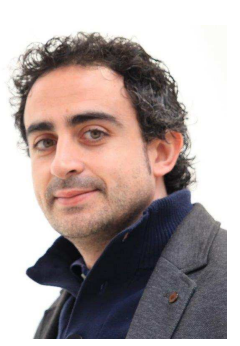

Mateo Iglesias received his Ms. Sc in Mobile Network Information and Communication Technologies (Telecommunication Engineering) from the University of Zaragoza (2011). He has been involved in $\mathrm{R} \& \mathrm{D}$ projects with companies and/or institutions in Energy Efficiency and Electromagnetic Compatibility (EMC) for communications, industry, scientific/medical and transportation applications since 2004. He has experience in collaboration with companies with a focus on training and EMI/EMC troubleshooting. Author/co-author of around 20 publications as a result of research activities.



Álvaro Pradas, Ms. Sc. in Electronics Engineering (2013), University of Zaragoza (Spain). Starting work at ITAINNOVA in 2010 in Electromagnetic Compatibility Laboratory. Since 2012 he belongs to the Power Electric Systems group where he takes part in $R \& D$ projects related to power electronics, energy conversion and efficiency from different sectors, including high energy physics, automotive and vertical transportation applications.

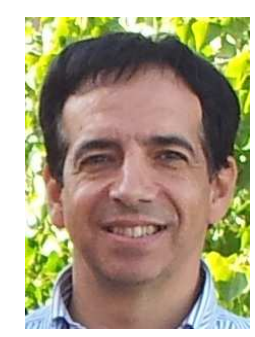

Javier Piedrafita, Industrial Engineer, University of Zaragoza (1993). His career has been mainly developed in the Instituto Tecnológico de Aragón (ITAINNOVA, Zaragoza, Spain) as a designer and developer of electronics systems, both in research projects and in industrial applications. He has worked in three main fields: embedded systems, functional safety and power electronics. He is currently working in the group of Electrical Systems at ITAINNOVA, where he has collaborated in several research projects: Windturbars (FP7, windturbines sector), DET4HEP (Spanish National Research Program 2011-2013, high-energy physics sector) and EVECTOORC (FP7, electric vehicle sector). 